\title{
An Optimized Computer Vision Approach to Precise Well-Bloomed Flower Yielding Prediction using Image Segmentation
}

\author{
Rupinder Kaur \\ M.Tech CSE (Student), \\ Jayoti Vidyapeeth Women's University, Jaipur, \\ Rajasthan, India
}

\author{
Shrusti Porwal \\ Assistant Professor,CSE Department \\ Jayoti Vidyapeeth Women's University, Jaipur, \\ Rajasthan, India
}

\begin{abstract}
The objective of this paper is to explore the various red colored Rose flowers recognition and yielding through prediction precision using segmentation. The yield concludes an excellent impression with the proper information for image prediction precision for a flower evacuation. The main concern is to detect and yield the blossom roses grown in cultivated land is estimated to amount and the terms; conditions, Luminescence and rose species produce flowers without changing any natural abnormality is to confirm. In dynamic technology, efficient cultivation requires a wide usage in yielding process where segmentation carries basic module in image extraction. The current study use the computerization techniques through thresholding to extract flower and Hue's color code Segmentation through Otsu Algorithm along with Morphological Filters to acquire the fine yielding of highly bloomed rose flowers from an digital snapshot. The procedure of recognition carried out for 230 images. This technique approaches a precise the recognizing, yielding and counting of rose flower at about $83.33 \%$ with overall accuracy.
\end{abstract}

\section{General Terms}

Image Processing, Pattern Recognition, Rose Flower, Algorithm, Floriculture.

\section{Keywords}

Image Segmentation, Otsu algorithm, Morphological Filter, Yield prediction precision, Flower Extraction.

\section{INTRODUCTION}

In India, floriculture has becomes a economic trade and source of income in the business market. The flower cultivation has been performed since the immemorial time but the floriculture has only been practiced in recent year in marketing. The usage in economic lead to ease in agricultural land, potted land, seed productivity and flower yielding. The cultivated of flower is extremely enormous [9] as the flowers have the array of color along with the numerous species in the worldwide. This enormous species, rose flower are well known for its more than 100 species in the world. As the species enlarges its extraction through computer vision declines. This is because every rose flower is highly recognized for its various distinct color, texture, shape and size. This feature varies from rose to rose. Through developing technology, computerization technologies to Yielding the different type of flower but precision and yielding of single species of rose flower is quite undetermined. Thus, with the high utility, Precision agriculture practice has been enhancing in technology. This agriculture precision lead to have growth in farming process, efficient in cultivation; production, quality improvement while reducing the cultivation cost. Thus, optimality gained in the yielding with minimum effort in business retailing.

In the agricultural field, rose is the highly perceived flower considering its favorably recognized species [7]. Each and every Roses bloom all through the different atmospheric conditions, this change lead to have Rose farming acquisition that carries around a one third section of economic and commercial growth in International market. The farming utilizes the entire yieldable flower in the agricultural land. The advance technique use right cutting flower, proper storage and fine cooling cell with transportation. This necessitates a proper knowledge consequently precision technology is highly recognized by the farmers for an efficient farming.

The rose cutting and yielding of highly bloomed flower and good quality of flower is recognized so that the business achieves a high pitch of development. Thus, the main purpose is to design a computerization technique that can recognize, identify, enumerate and estimate the features extraction [1][4] and yielding technique.

\section{LITERATURE REVIEW}

Segmentation is used for extracting the object from its image through separating it from its foreground to foreground. This distinction is carried out through distinct feature of an object that considered being a fundamental property [2][5] of object. Through number of recognizing species, it's very challenging to precise and identifies every species of flower especially when the species is needed to be considered for Rose flower. Many researchers had purposed numerous automated systems for recognizing flower [3][14]. The Rose flower is practiced through automated harvesting [6] using computer technology. The yielding mapping and precision farming carries a highly significant improvement through prediction yielding system. The object yielding is accomplished through segmentation using feature extraction. The color is considered to be the most precise feature [12] for extraction and detecting any object form a digital image. The RGB as well as HSV are known to be considered a valuable approach for human perception [10].

Takesh Saitosh el al [8] carried a work on the blooming flower recognition through digital camera to extract the boundaries of flower region and achieves a minimal sun of local cost by route length. The GrabCut methodology is implemented for flower classification system extraction through loosely drag rectangular using alpha matting purposed by Carsten Rother el al [11]. Madirakshi Das el al [13] worked on Indexing of flower patent image with specialized dataset using spatial domain knowledge iterative segmentation algorithm for precise object precision. 


\section{MATERIAL AND TOOL}

To evaluate the work, the digital image of rose flower is captured. The camera is positioned $1.62 \mathrm{~m}$ above the top of the flowers and viewed the same positioned at every plot of where flower needed to be detected. The camera operator doesn't enter in the flower cultivated land as to avoid damage to its plant. The digital camera having 13 mega pixel capacity from which image produce of $4608 \times 2592$ pixel dimension. At the time of image capturing, the camera should be statically still. Image that was downloaded from camera to personal computer is compatible of JPEG Format.

Further, work implementation was carried out on Core2 duo $2.20 \mathrm{GHz}$ with $3.00 \mathrm{~GB}$ RAM. The image processing and segmentation analysis is performed through MATLAB R2013a software that carried out 230 image testing. The new version of software and high computational power provides an efficient performance for testing and utility of result.

Furthermore, the yielding prediction precision is preprocessed initial by re-sizing the image, color preserving etc. For the work implementation, firstly the image is captured through digital camera and pre-processing is applied on the various sampled flower shown in fig [1]. After this, color of image is preserved using Hue's color code segmentation and carried out a HSV space. The color space is described as a default image consideration as to be a RGB, CIE L*a*b*, perception uniformity [15] of color space. This color feature is considered to be main phenomena for counting and yielding flower from the field, if this factor is not considered then result utility will be dismissed.
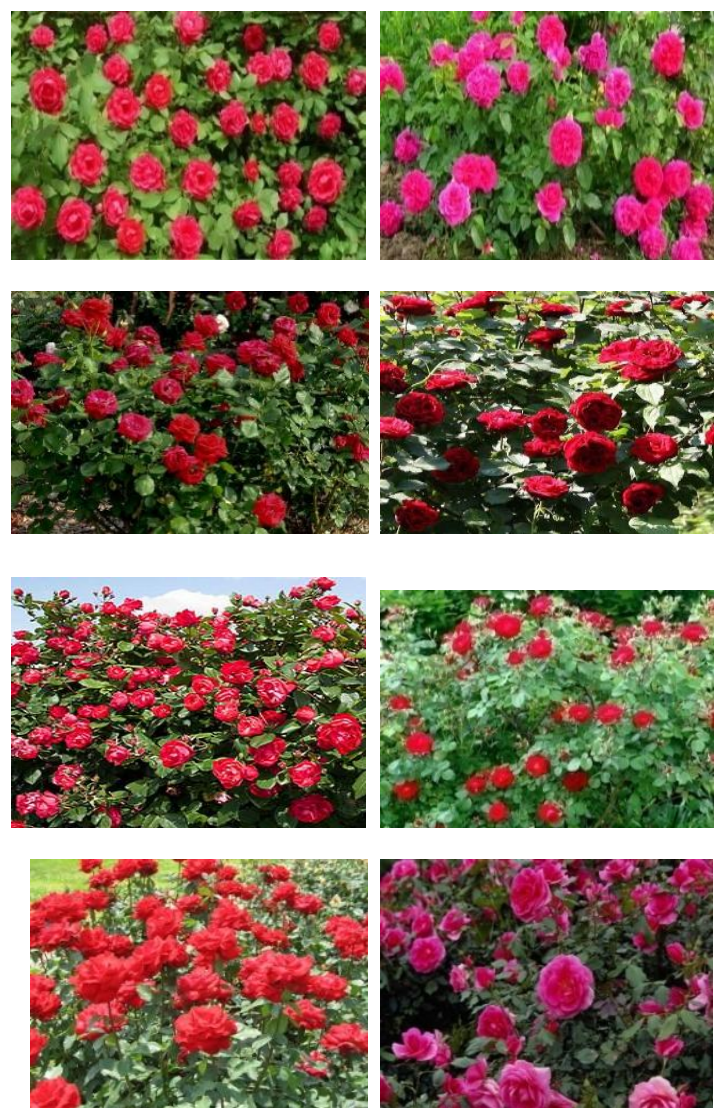

Fig 1: Sample Flowers Image

\section{METHODOLOGY}

Using computerization technique, the image recognition and counting of rose flower should be accomplished to perform accurately and flower which needed to perceive should be well bloomed. The structural framework of system is mentioned in fig [3]. The distinct layout is allocation of system procedure

and work manipulation. This yielding occurred through capturing image, color preservation through various features extraction, digititilaztion through filters and morphology technique and finally yielding the counted flower in the image represented in figure shown in fig 2.

After capturing the rose image, the color which needed to be extracted is preserved using Hue's Color code segmentation. The image containing RGB is transform into HSV Color to fetch the H-Component. Thus, region of flower is recognized through color features. Below in Fig 3, the Hue's color Circle is given where all the shades of colored are marked.

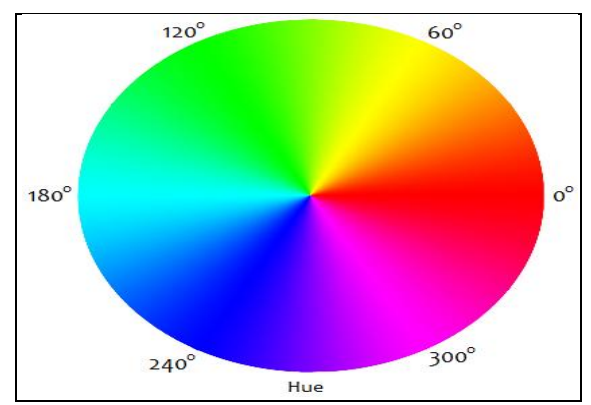

Fig 3: Hue Color Space

The Various colors are segmented with its color ranges and evaluate the feature extracting of object form its image using this color ranges. These colors are used to discriminate various objects with primarily color where image retival uses the color feature which are the most widely used feature [16] and color image segmentation [17].

Thus, the Table 1 represents the range of the primary contributed colors [12] that are helped to determine the color range implementation in the paper work and utilized for improved accomplishment of work.

Table 1: Color Range

\begin{tabular}{|cc|}
\hline Angle & Color \\
\hline $0-60$ & Red \\
\hline $60-120$ & Yellow \\
\hline $120-180$ & Green \\
\hline $180-240$ & Cyan \\
\hline $240-300$ & Blue \\
\hline $\mathbf{3 0 0 - 3 6 0}$ & Magenta \\
\hline
\end{tabular}




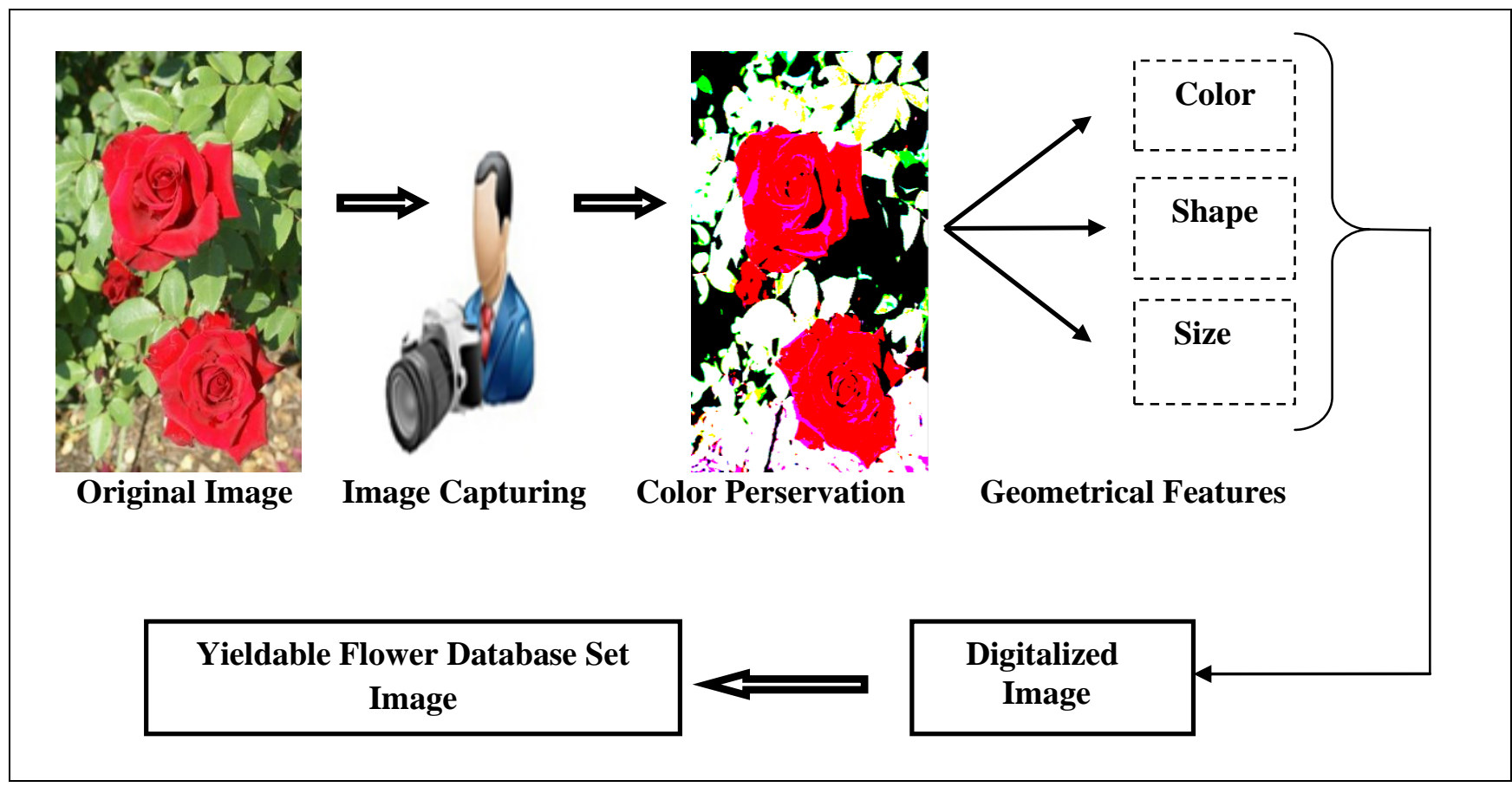

Fig 2: System Architecture

\subsection{Flower Extraction}

Yielding and recognition of flower from its background is executed using image segmentation through Otsu thresholding. This thresholding extract the region of object from an image. The grayscale image is segmented as basically, distinct ranges are describe across a gray level known to be threshold

$$
\mathrm{I}(\mathrm{x}, \mathrm{y})= \begin{cases}0 & \mathrm{I}(\mathrm{x}, \mathrm{y})<T \\ 1 & \mathrm{I}(\mathrm{x}, \mathrm{y}) \geq \mathrm{T}\end{cases}
$$

Where $\mathrm{T}$ is the threshold having some constant vale where pixel is replaced accordingly and $\mathrm{x}, \mathrm{y}$ are the are merged to form a unit of pixels. The value, I constitute of grey scaled range of image. Thus, to calculate the color code of HSV coded as Hue along with Saturation and also the Value that features as:

$$
\mathrm{P}_{\mathrm{i}}=\left\{\mathrm{P}_{1}, \mathrm{P}_{2}, \mathrm{P}_{3} \ldots \mathrm{P}_{\mathrm{n}} \mid \mathrm{P}_{\mathrm{i}}(\mathrm{H}, \mathrm{S}, \mathrm{V})\right.
$$

This pixel of class are represented as flower extraction and carries a HSV color code using range given in Table 1.

The color code of all HSV color is separately define using equation (1) and (2)

$$
\begin{aligned}
& \mathrm{I}_{\mathrm{H}}(\mathrm{x}, \mathrm{y})= \begin{cases}0 & \mathrm{PH}_{\mathrm{H}}<T \\
1 & \mathrm{PH}_{\mathrm{T}}\end{cases} \\
& \mathrm{I}_{\mathrm{S}}(\mathrm{x}, \mathrm{y})= \begin{cases}0 & \mathrm{Ps}<T \\
1 & \mathrm{Ps}_{\mathrm{S}} \mathrm{T}\end{cases}
\end{aligned}
$$

This thresholding is done through computerized technique through distinct range allotment of various different flowers. We selected a rose flower thresholding to analyse the HSV of flower image.

\subsection{Morphology}

Morphology is a process that rely the ordering pixel value associated with its shape also with features of an image. The morphology technique is applied on a input image for converting it to a structural small size output image. The neighbored pixels are matched with relatively adjacent pixel to form a possible structuring image. In image, these arranged elements are represented as 1 or 0 and form a pixel matrix through a minor image of binary. The geometrical morphology is applied to prepossess the planer gray scale image through lattice theory, set theory and random functions. The functional transformation is characterized on the geometrical structure as shape, size and geodesic distance. The morphology operation is represented in image through set theory. Let an image is represented as $\mathrm{a}(\mathrm{x})$ and structuring function(x). This representation is applied to grey scaled image dilation on a function $\mathrm{a}$ in $\mathrm{b}$ as:

$$
\begin{aligned}
& (\mathrm{a} \oplus \mathrm{b})(\mathrm{x})=\bigcup_{y \in E}[a(y)+b(x-y) \\
& (\mathrm{a} \Theta \mathrm{b})(\mathrm{x})=\bigcap_{\mathrm{y} \in \mathrm{E}}[\mathrm{a}(\mathrm{y})-\mathrm{b}(\mathrm{x}-\mathrm{y})]
\end{aligned}
$$

In gray scaled image, morphology evolves basic operation as Euclidean Distance, Erosion as well as including Dilation. The morphology filters are highly used for image extraction in grey scaled images and perceive accuracy using segmentation. This filter makes image compressive in nature through which size for object recognizability of image becomes more perspicuous. Thus, this usage makes the flower image more recognizable and perceivable.

If any change in their shape and size lead to have a faulty resultant in computation technique but the image enhancement minimizing their type of problems and yield an optimal result to identify every object without missing a single one in the image. This carries a highly progressive acquiring resultant in image prediction and precision. 


\subsection{Optimized Flower Yielding Algorithm}

Step1: Compute the initialization of image capturing $\mathrm{I}_{\mathrm{i}}$.

Step 2. Resize the image Ir with proper dimension.

Step 3. Convert RGB color image to Gray scale Image $I_{(x, y)}$.

$$
\mathrm{I}_{(\mathrm{x}, \mathrm{y})} \leftarrow \mathrm{I}_{\mathrm{r}}
$$

Step 4: Implement threshold technique for HSV color Code as.

$$
\begin{aligned}
& \mathrm{I}_{\mathrm{H}}(\mathrm{x}, \mathrm{y})= \begin{cases}0 & \mathrm{I}(\mathrm{x}, \mathrm{y})<T \\
1 & \mathrm{I}(\mathrm{x}, \mathrm{y}) \geq \mathrm{T}\end{cases} \\
& \mathrm{I}_{\mathrm{S}}(\mathrm{x}, \mathrm{y}) \quad= \begin{cases}0 & \mathrm{I}(\mathrm{x}, \mathrm{y})<T \\
1 & \mathrm{I}(\mathrm{y}, \mathrm{y}) \geq \mathrm{T}\end{cases} \\
& \mathrm{I}_{\mathrm{V}}(\mathrm{x}, \mathrm{y}) \quad= \begin{cases}0 & \mathrm{I}(\mathrm{x}, \mathrm{y})<T \\
1 & \mathrm{I}(\mathrm{x}, \mathrm{y}) \geq \mathrm{T}\end{cases} \\
& \mathrm{I}(\mathrm{x}, \mathrm{y}) \leftarrow \mathrm{I}_{\mathrm{H}}(\mathrm{x}, \mathrm{y}) \cup \mathrm{I}_{\mathrm{S}}(\mathrm{x}, \mathrm{y}) \cup \mathrm{I}_{\mathrm{V}}(\mathrm{x}, \mathrm{y})
\end{aligned}
$$

Step 5: Object extraction of a particular color range of code:

$$
\text { If }\left(\mathrm{I}(\mathrm{x}, \mathrm{y}) \geq \mathrm{I}_{\text {range }} \| \mathrm{I}(\mathrm{x}, \mathrm{y}) \leq \mathrm{I}_{\text {range }}\right) \text { then }
$$

Object Extract from Boundary Of Image

Else Goto Step 3.

Step 6: Noiseless Greyscale image from extracted image.

Step 7: Apply morphological filter on extracted Flowers to convert into small size.

Step 8: Convert to RBG after counting and yielding Flower. Step 9: else, Goto Step 2.

Step 10: Output Yield Image Recognized.

Algorithm: Flower Boundary Tracing and Yielding

\subsection{Matlab Implementation}

For flower yielding and recognizing, various steps are evolved and manipulated in Matlab software. This help to retrieve a finite resultant yielding through image segmentation .Furthermore, fine extraction is determined for rose flower counting. The flower features is extracted from binary image and individual flower detection is yield through Matlab implementation using image processing

\section{EXPERIMENTAL RESULT}

The system conducted the experimental test on 230 flowers with numerous unlike distinct species of flower with combining the rose flowers. The fine resultant system performance and efficiency is gained through detection moreover the counting of well-bloomed flower for cutting and ready to cut-through acquires overall efficiency of $83.33 \%$.

The work accomplishment achieved through recognizing the flowers, demarcating, and masterly yielding of flower through black circled flower with exterior area where the rose flower is acquired. Furthermore the system counts the number of flower which has been yielded. Some contaminated result occurrences due to overlapping and edge-cutted flower at the time of capturing Image.

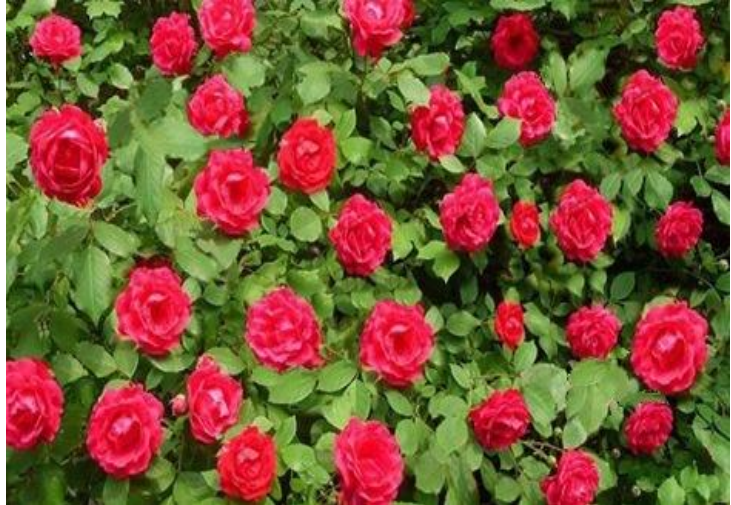

Original Image

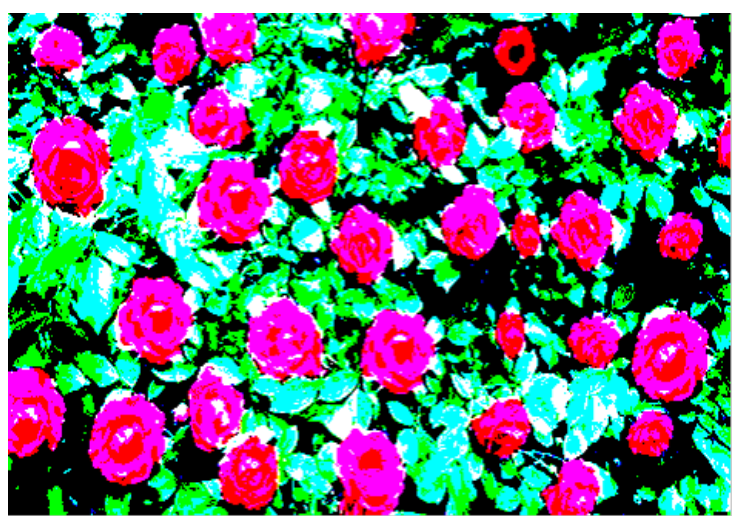

Color Preservation

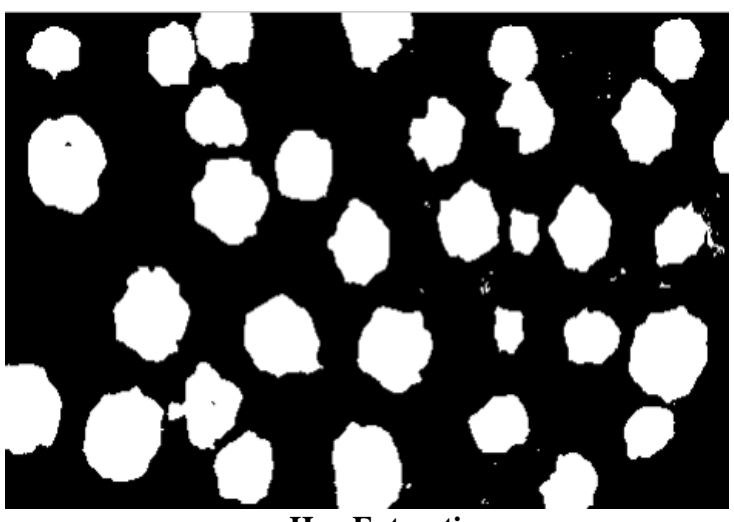

Hue Extraction

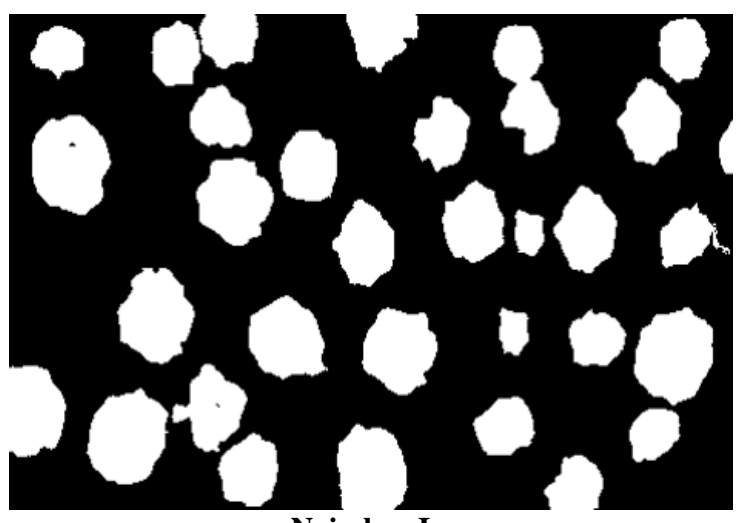

Noiseless Image 


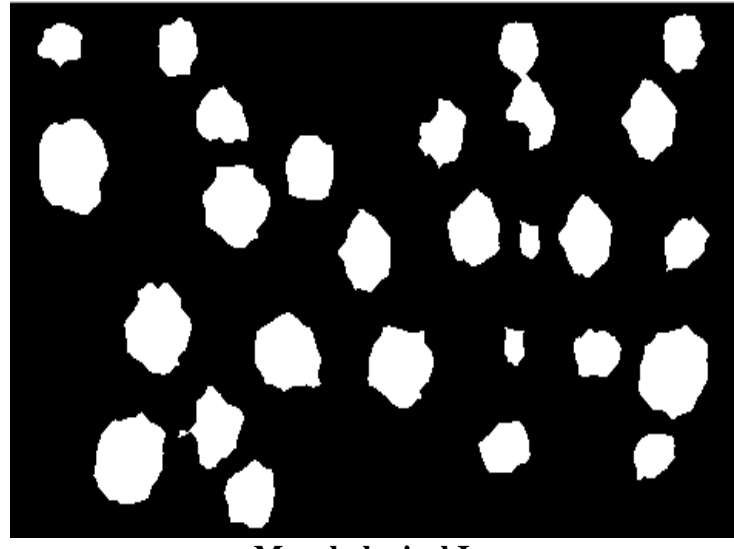

Morphological Image

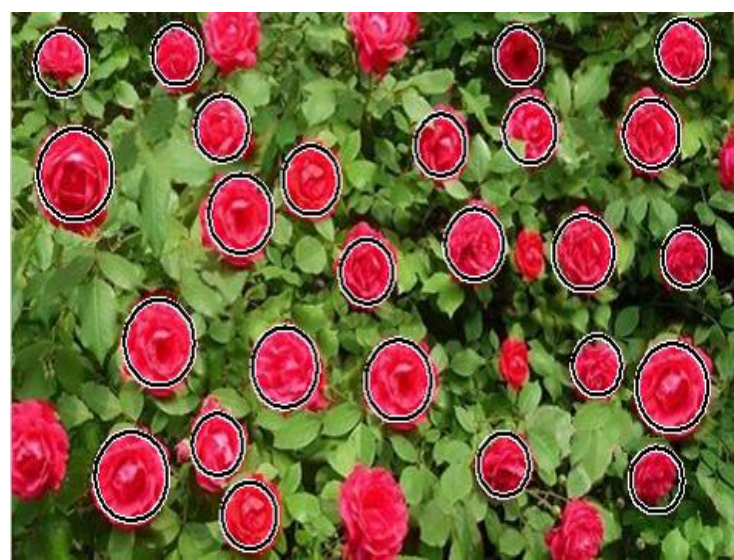

Flower Yielded Image

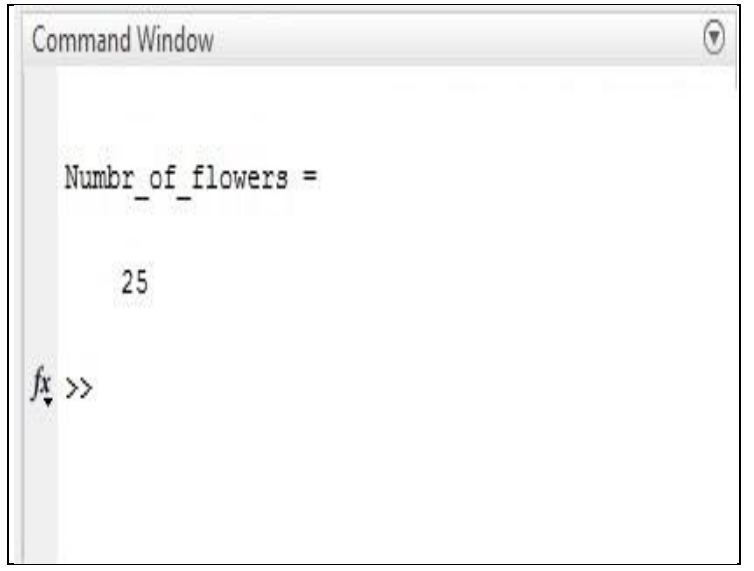

System Counted Yield Flower

\section{CONCLUSION}

In this research, the system is adequate to achieve the main objective by extracting and counting the well bloomed flowers through fine reorganization computer vision. Thus, computerized approach to optimize the performance and productive capacity of system acquires an effective yielding. This proposed methodology surpass all comparative state of art as well as algorithm flower yielding illustrates exemplary in counting of flower objects. The proposed work expected to inspire the new thinking and move toward a new way where the rose flower production is moved in higher qualities from which demanding of rose cut-flower in international market is its highest pitch. This flower yielding and counting will achieves the highest utility in agricultural rose farming including the floriculture to work preferably much superior for future exertion. This present methodology suffers from some condition that forwarded the result affected via a distance variation, capturing problem for full acquire flower in image, illumination which needed to be study further for addressing this limitations. The developing technology and techniques will acquires more improved methodology for precision yielding of rose flowers.

\section{REFERENCES}

[1] M. Zhenjiang, M.H. Gandelin, and Y. Baozong, "Fourier Transform-Based Image Shape Analysis and Its Application to Flowers Recognition", International Conference on Signal Processing, Beijing, China, 26-30 pp. 1087-1090.

[2] Anil Chitade,Dr. S.K. Katiyar,"Colour Based Image Segmentation Using K-Means" Clustering,01/2010.

[3] C. Pornpanomchai, and A. Suppaiboonvong, "Thai Blooming Flower Recognition", International Joint Conference on Computer Science and Software Engineering, Phuket, Thailand, 13-15 May 2009, pp. 219-224.

[4] J. Zou, and G. Nagy, "Evaluation of Model-Based Interactive Flower Recognition", in Proceeding of International Conference, Cambridge, 23-26 August 2004, pp. 311-314.

[5] Ing-Sheen Hsieh, Kuo-Chin Fan , "Color image retrieval using shape and spatial properties," Pattern Recognition, 2000. Proceedings. 1th International Conference on, vol.1, no., pp.1023-1026 vol.1, 2000.

[6] Baykan et al, Case study in effects of color spaces for mineral identification, Scientific Research and Essays Vol. (11), pp. 1243-123, 4 June, 2010.

[7] Rupinder Kaur,"E Yield Prediction Precision of Rose Flower Recognition using Segmentation", International Journal of Engineering Technology and Computer Research; Volume 3; Issue 2; Page No. 31-33.

[8] Saitoh, T., Aoki, K., Kaneko, T., "Automatic recognition of blooming flowers," Pattern Recognition, 2004. ICPR 2004.

[9] Rupinder Kaur, Barkha Malkaniya, Ms. Shrusti, "A Survey of Image Segmentation of Color Flower Yield Prediction Precision", International Journal of Innovative Computer Science \& Engineering, Vol. 2; Issue 2; Page No.01-04.

[10] Christopher Henry and James F. Peters,"Perception-basedimage classification"; ISSN: 1756-378X, 2008.

[11] Carsten Rother, Vladimir Kolmogorov and Andrew Blake, "GrabCut - Interactive Foreground Extraction using Iterated Graph Cuts", ACM Transaction on Graphic; vol 23 Iss. 3; PPg: 309-314.

[12] Baykan et al, Case study in effects of color spaces for mineral identification, Scientific Research and Essays Vol. (11), pp. 1243-123, 4 June, 2010.

[13] Das M.; Manmatha R.; Riseman E.M.; "Indexing flower patent images using Domain knowledge," IEEE Intell Syst 14:24_33, 1999.

[14] M.E. Nilsback, and A. Zisserman, A Visual Vocabulary for Flower Classification, IEEE Computer Society Conference on Computer Vision and Pattern 
Recognition, New York, USA, 17-22 June 2006, pp. 1447-1454.

[15] Albuz el al. 2000. Quantized CIE L*a*b* Space and Encoded Spatial Structure for Scalable Indexing of Large Color Image Archives. 2000 IEEE Conference on Acoustics, Speech, and Signal Processing, 4:1995-1998.
[16] Hong An-xiang, CHEN Gang, LI Jun-li, CHI Zhe-ru, ZHANG Dan,"A flower image retrieval method based on ROI feature", 2004(7):764-772

[17] Yining el al., Color Image Segmentation.IEEE Computer Society Conference on Computer Vision and Pattern Recognition, 2:445-451. 